\title{
Optical tuning of the charge carrier type in the topological regime of InAs/GaSb quantum wells
}

\author{
G. Knebl, ${ }^{1}$ P. Pfeffer, ${ }^{1}$ S. Schmid, ${ }^{1}$ M. Kamp, ${ }^{1}$ G. Bastard,${ }^{2}$ E. Batke, ${ }^{1}$ L. Worschech, ${ }^{1}$ F. Hartmann,,${ }^{1, *}$ and S. Höfling ${ }^{1,3}$ \\ ${ }^{1}$ Technische Physik, Physikalisches Institut and Röntgen Center for Complex Material Systems (RCCM), Universität Würzburg, \\ Am Hubland, D-97074 Würzburg, Germany \\ ${ }^{2}$ Laboratoire Pierre Aigrain, École Normale Supérieure, PSL Research University, CNRS, Université Pierre et Marie Curie, \\ Sorbonne Universités, Université Paris Diderot, Sorbonne Cité, 24 rue Lhomond, 75005 Paris, France \\ ${ }^{3}$ SUPA, School of Physics and Astronomy, University of St Andrews, St Andrews KY16 9SS, United Kingdom
}

(Received 15 March 2018; revised manuscript received 14 May 2018; published 16 July 2018)

\begin{abstract}
We study the optical tunability of the charge carrier type in InAs/GaSb double quantum wells with its type-II broken band alignment and inverted band structure. Under constant optical excitation, the majority charge carrier type switches from electron to hole. Within the majority charge carrier type transition, the coexisting minority charge carrier contribution indicates electron-hole hybridization with a nontrivial topological insulating phase. The optical tuning is attributed to the negative photoconductivity of antimonide materials in combination with a persistent charge carrier buildup of photogenerated charges at the surface and substrate side of the device, respectively. Our study of the tuning of an InAs/GaSb double quantum well heterostructure reveals that an electro-optical switching is possible and paves the way to an optical control of the phase diagram of InAs/GaSb topological insulators.
\end{abstract}

DOI: 10.1103/PhysRevB.98.041301

Topological insulators (TIs) are a state of matter, characterized by an insulating bulk but gapless helical surface or edge states [1-4]. In 2007, the first successful experimental demonstration was realized in $\mathrm{HgTe} / \mathrm{CdTe}$ quantum well heterostructures, when König et al. [5] provided experimental evidence of two propagating edge states leading to a quantized conductivity of $2 e^{2} / h(e$ : elementary charge; $h$ : Planck constant) in the topological regime. For this particular material system, a trivial insulating regime is present below a critical HgTe layer thickness $d_{\text {crit }}$ and the topological insulator regime with its inverted band alignment is observed for quantum well thicknesses exceeding $d_{\text {crit }}$. Hence, the transition between the two regimes depends on the quantum well thickness. This, however, limits the device variability and tunability via external control parameters as the quantum well thickness is adjusted in the epitaxial growth process. Such device variability and tunability via external electric fields is of key importance for exploitation in devices, which is offered by the predicted topological insulator based on $\mathrm{InAs} / \mathrm{GaSb}$ double quantum wells (DQWs).

InAs/GaSb DQWs have a type-II band alignment in which the hole and electron states are spatially separated and mainly localized in the GaSb and the InAs layers, respectively. Indeed, it was predicted that this material system could be a topological insulator [6]. The alignment between the electron and hole state in this system can be controlled by external electric fields, making it, in principle, possible to tune between the topological insulating phase, i.e., hole states are energetically above the electronic states, and the trivial insulating phase, when the electron state is above the hole state. Despite many

\footnotetext{
${ }^{*}$ Corresponding author:

fabian.hartmann@physik.uni-wuerzburg.de
}

investigations [7-21], a fully convincing demonstration of helical edge states in the topological insulating phase in this material system is still elusive, although the tunability between normal to topological nontrivial insulating phases was proven with a dual gated approach [14]. Due to the peculiarities of the so-called 6.1 ̊ material system (InAs, GaSb, and AlSb), e.g., the Fermi level pinning of InAs in the conduction band [22-24], additional parasitic surface conducting properties of oxidized antimonides [25-27], and the leakage currents of the back gate via defect states [28] make it difficult to analyze transport phenomena in InAs/GaSb DQWs and to observe topological nontrivial phases via a dual gating approach.

In this study, we utilize optical excitation to show that the transport regime of $\mathrm{InAs} / \mathrm{GaSb}$ double quantum wells can be tuned from electronlike to holelike without needing any external electric fields. For constant optical excitation and short illumination times, the transport is dominated by electrons, while for long illumination times the transport is dominated by holes. At an intermediate value of illumination time - it is in the switching area - both carrier types are present simultaneously, indicating electron-hole hybridization with a nontrivial topological insulating phase. The results of optical gating are similar to the data shown for the dual electrical gating approach [14]. The optically induced dual gating of InAs/GaSb double quantum wells may amend for leakage currents in this material system and pave the way toward electro-optical applications utilizing the topological insulating phase in InAs/GaSb double quantum wells.

Figure 1(a) shows an electron microscopy image of the studied heterostructure with the InAs/GaSb double quantum well residing about $50 \mathrm{~nm}$ below the surface. The heterostructure was grown by molecular beam epitaxy on an $n$-doped GaAs substrate with $\mathrm{GaSb}$ quasisubstrate [29]. For growth details, see the Supplemental Material [30]. The simulated valence 

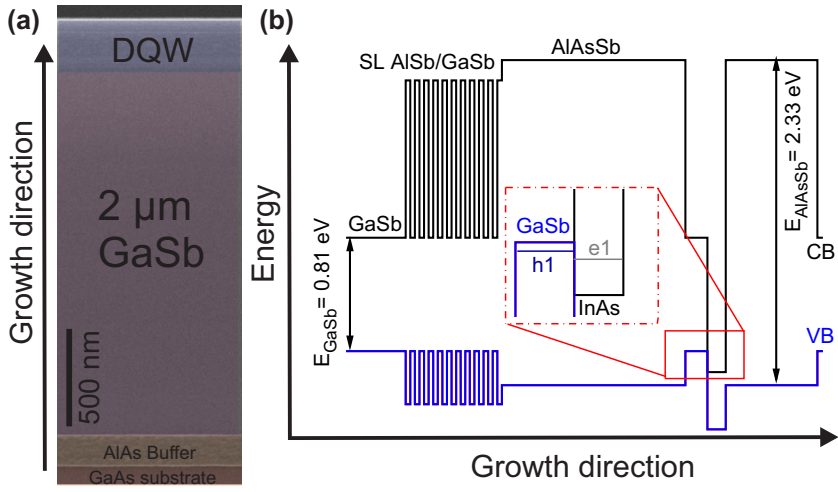

FIG. 1. (a) Scanning electron microscopy image of the full heterostructure. The active quantum well region consists of the InAs/GaSb DQW embedded between two $\mathrm{AlAs}_{0.11} \mathrm{Sb}_{0.89}$ barriers. The quantum well thicknesses are 10 and $12 \mathrm{~nm}$ for GaSb and InAs, respectively. (b) Numerical simulation of the valence and conduction band profile of the heterostructure, including the GaSb quasisubstrate, the $\mathrm{AlSb} / \mathrm{GaSb}$ superlattice (SL), and the AlAsSb/GaSb/InAs/AlAsSb region with its type-II band alignment between InAs and GaSb. The localized electron $\left(e^{-}\right)$and hole $\left(h^{+}\right)$states are depicted in the zoom in the active region. $\mathrm{CB}$ and $\mathrm{VB}$ denote the conduction and valance band, respectively.

and conduction band profiles of the studied heterostructure are shown in Figure 1(b) [31]. The InAs and GaSb quantum well thicknesses (12 and $10 \mathrm{~nm}$ ) are chosen, to ensure band inversion between the hole level $\left(h^{+}\right)$, residing on the $\mathrm{GaSb}$ side of the DQW, and the electron level $\left(e^{-}\right)$, residing on the InAs side of the DQW. From the numerical simulation, the energy difference between the hole and electron level is calculated to be $\approx 18 \mathrm{meV}$ at the $\Gamma$-point and the hybridization opens a gap at $k_{\text {cross }} \approx 0.1 \mathrm{~nm}^{-1}$. After the epitaxial growth process, a standard Hall bar layout was fabricated. The Hall bar dimensions used within this study are $20 \mu \mathrm{m}$ by $60 \mu \mathrm{m}$ (width, length) and, if not stated explicitly otherwise, all measurements presented in this study were obtained at $4.2 \mathrm{~K}$. For the optical illumination, two light-emitting diodes were used that emit photons with energy $1.80 \mathrm{eV}$ (Red-LED) and $1.32 \mathrm{eV}$ (IR-LED). The photon energies used in this study are below the band-gap energy of the surrounding $\mathrm{AlAs}_{0.11} \mathrm{Sb}_{0.89}$ barrier $\left[E_{\text {gap }} \approx 2.37 \mathrm{eV}\right.$; see Fig. 1(b)] but well above the band-gap energy of GaSb [ $E_{\text {gap }} \approx 0.81 \mathrm{eV}$; see Fig. 1(b)].

Figure 2(a) shows the Hall resistance $R_{x y}$ (black) and longitudinal resistance $R_{x x}$ (blue) as a function of illumination time, recorded for a constant applied magnetic field of $B=2 \mathrm{~T}$. For the depicted measurement, the Red-LED was switched on at $t=0 \mathrm{~s}$ and the Hall bar was illuminated permanently up to $t=70 \mathrm{~s}$ at a constant Red-LED light power. During the illumination time, $R_{x x}$ and $R_{x y}$ were recorded simultaneously. Initially and without optical excitation (i.e., at $t=0 \mathrm{~s}$ ), the value of the longitudinal resistance is $R_{x x}=7.5 \mathrm{k} \Omega$. The positive Hall resistance indicates that electrons are the majority charge carriers. Under constant optical excitation, $R_{x x}$ increases and peaks at an illumination time around $t \approx 28 \mathrm{~s}$ with a maximal resistance value of $R_{x x, \max }=33.5 \mathrm{k} \Omega$. Longer light exposure times lead to a reduction of the longitudinal resistance until $R_{x x}$ reaches a saturation value of about $R_{x x}=14.5 \mathrm{k} \Omega$ at

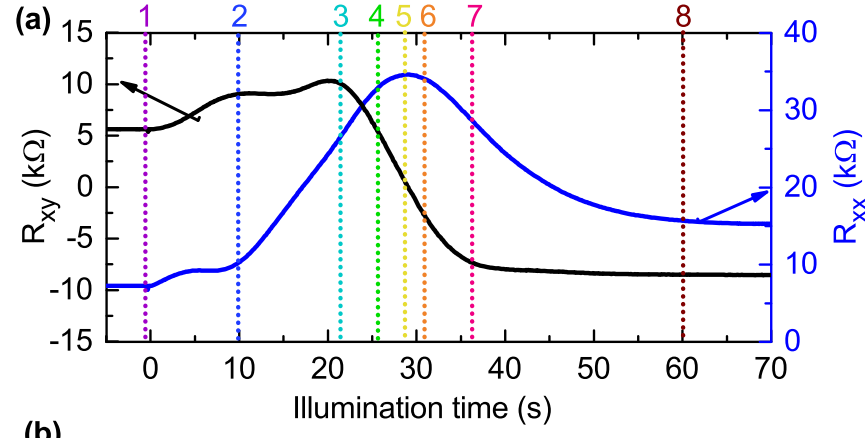

(b)

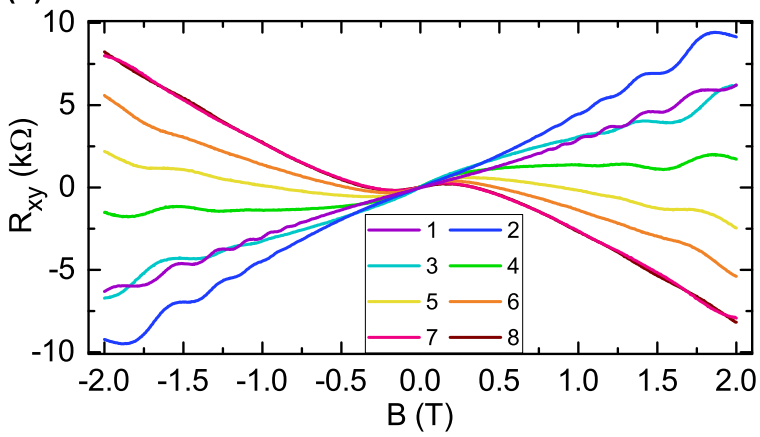

FIG. 2. (a) $R_{x y}$ (black solid line) and $R_{x x}$ (blue solid line) versus the illumination time of the Red-LED, recorded at a constant magnetic field of $2 \mathrm{~T}$ and $T=4.2 \mathrm{~K}$. $R_{x x}$ peaks with a maximum resistance $R_{x x, \max }=33.5 \mathrm{k} \Omega$ at $t \approx 28 \mathrm{~s}$, at which the Hall resistance $R_{x y}$ depicts a sign reversal from positive to negative values. Below and above the illumination time $t \approx 28 \mathrm{~s}$, the majority charge carrier switches from electrons to holes. (b) Hall resistance versus the magnetic field, measured for the illumination times indicated in the vertical lines and corresponding numbers from panel (a). The Hall resistance traces are color coded. In the electron regime, corresponding to low illumination times, the Hall resistance curves show well pronounced integer quantum Hall plateaus. In the hole regime, corresponding to high illumination times, the Hall resistance shows distinct two-carrier transport with a sign reversal of the Hall resistance at about $B=0.5 \mathrm{~T}$.

$t=70 \mathrm{~s}$ (end of the light exposure). The corresponding Hall resistance measurement shows that a sign reversal of the Hall resistance from $t=0$ to $70 \mathrm{~s}$ occurs and that the majority charge carrier type switches from electrons to holes. This transition with a vanishing Hall resistance occurs at the peak position of the longitudinal resistance, i.e., at an illumination time of $t \approx 28 \mathrm{~s}$. The maximal longitudinal resistance was also measured for different temperatures and fitted by an Arrhenius function $1 / R_{x x ; \max } \propto \exp \left(-E_{\mathrm{gap}} / 2 k_{B} T\right)$ [11,18]. For temperatures down to $10 \mathrm{~K}$ we derive $E_{\text {gap }}=11.0 \mathrm{meV}$ and $T_{\text {gap }}=128 \mathrm{~K} . E_{\text {gap }}$ compares well to the inversion gap of $18 \mathrm{meV}$. Additional data is shown in the Supplemental Material [30]. After the light was switched off at the $R_{x x \text {, max }}$ value, the value was stable even after 18 hours. To switch back to the initial longitudinal resistance value after light exposure, tens of microwatt electrical power is needed (for at least $10 \mathrm{~min}$ ) to restore the initial value.

Figure 2(b) shows magnetic-field-dependent Hall resistance measurements as obtained for eight illumination times as indicated in Figure 2(a) (labels 1-8, color coded). Prior to each magnetic-field measurement, the sample was illuminated 
for a fixed amount of time, e.g., $10 \mathrm{~s}$ for point 2 . Then, the illumination was switched off and the Hall resistancemagnetic-field measurement was performed. After the measurement, the LED was switched on again, e.g., $12 \mathrm{~s}$ for point 3 (in total: $22 \mathrm{~s}$ exposure time), and the next Hall resistance-magnetic-field measurement was performed. For short illumination times (points 1 and 2), the Hall resistance shows well pronounced integer quantum Hall plateaus at multiples of the resistance quantum $R_{k}=h / e^{2}$. Although the Hall resistance traces seem to evolve perfectly linear at low magnetic fields, a weak nonlinear slope is observed that indicates already the existence of a minority hole density. For points 3 and 4 , slightly left-handed to the $R_{x x \text {, max }}$ peak position at $t \approx 28 \mathrm{~s}$, the Hall resistance becomes evidently nonlinear at low magnetic fields and is well reduced compared to Hall resistance values obtained at shorter illumination times (points 1 and 2). This indicates that although the majority charge carriers are electrons, a non-negligible minority hole carrier density starts to evolve. For illumination times around the peak position of $R_{x x}$ (points 5 and 6), the Hall resistance changes sign and is positive below magnetic-field values of $1 \mathrm{~T}$ (point 5) and $0.5 \mathrm{~T}$ (point 6), and negative above $B>1 \mathrm{~T}$. The majority charge carrier type switched to holes. For longer light exposure times (points 7 and 8), the Hall resistance remains nonlinear with a negative and positive Hall resistance at large and low magnetic fields, respectively. For even larger illumination times (not shown here), the nonlinearity of the Hall resistance remains and never reaches a simple linear trace as a signature of hole transport alone. The apparent nonlinearity of the Hall resistance is caused by the coexistence of electrons and holes.

Figures 3(a) and 3(b) depict the charge carrier densities and mobilities of both charge carriers, as obtained from a two-carrier model according to Ref. [19], respectively. The results show the mainly $n$-type conduction before the $R_{x x}$ peak with an electron carrier density in the $10^{11} \mathrm{~cm}^{-2}$ range, whereas afterward the $p$-type carrier density outweighs the $n$-type density. The charge carrier densities within the hole and electron regime (well above and below $R_{x x, \max }$ ) are comparable. The significantly reduced mobility of holes compared to electrons (about a factor of 4), leads to the observable integer quantum Hall plateaus in the electron regime [see points 1 and 2, Fig. 2(b)], the nonlinear Hall resistance in the electron regime with minority hole carrier densities, and the sign reversal of the Hall resistance in the hole regime. The electron mobilities in the hole-dominated regime are $\sim 35 \times 10^{3} \mathrm{~cm}^{2} / \mathrm{V} \mathrm{s}$ and the hole mobilities in the electron-dominated regime are $\sim 10 \times 10^{3} \mathrm{~cm}^{2} / \mathrm{V}$ s [both not shown in Fig. 3(b)]. Compared to mobility values reported in the literature, the electron mobility presented here is about one order of magnitude below reported values (see Refs. [14,28]), but the hole mobility compares well with other literature values [14]. Record mobilities reported in the literature were, however, obtained in heterostructures grown on $\mathrm{GaSb}$ substrates.

Under light exposure with the Red-LED, the charge carrier type can be switched from electrons to holes. At an illumination time of $t \approx 28 \mathrm{~s}$, the Fermi energy lies within the gap and the longitudinal resistance becomes maximal. The illumination time depends on the light intensity, and optical tuning speed can be controlled over a broad time range. Figure 4(a) displays
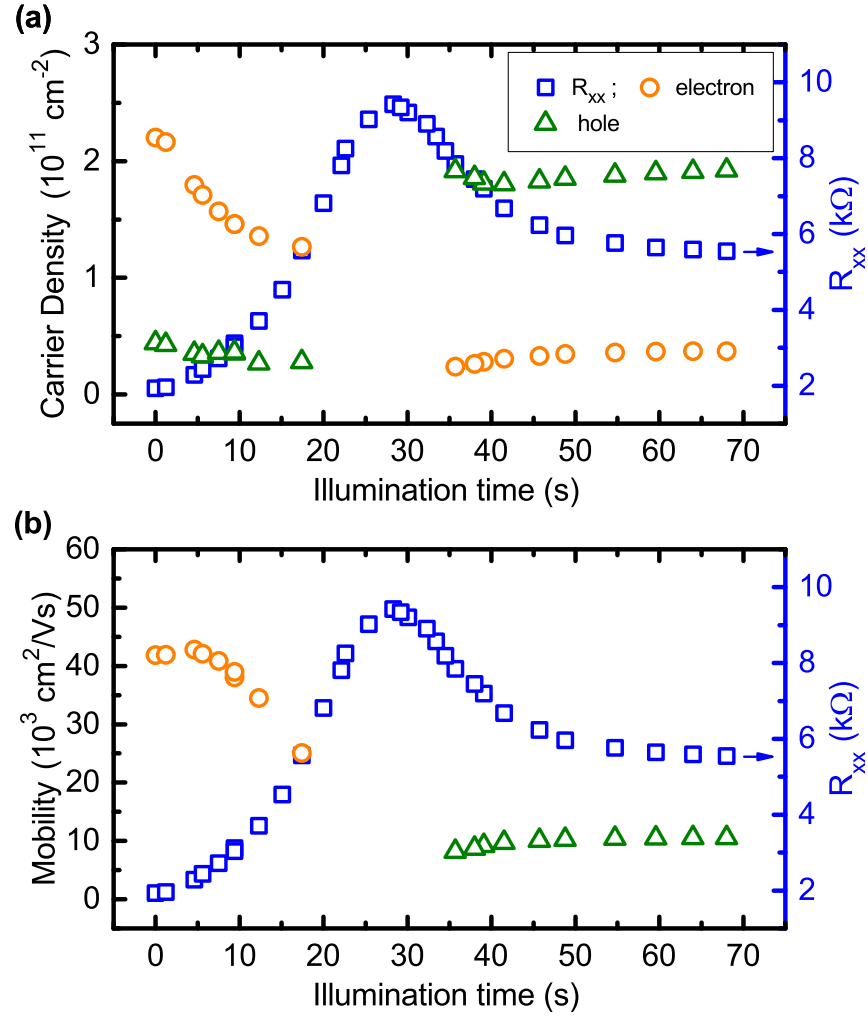

FIG. 3. Charge carrier densities [in (a)] and mobilities [in (b)] of the electron and hole gases as derived from a two-carrier model versus the illumination time. For comparison, $R_{x x}$ (blue squares) for $B=0 \mathrm{~T}$ is also shown.

that the required illumination time to reach $R_{x x, \max }$ can be reduced, if the Red-LED light power is increased. For the depicted measurement and for each light power, the Red-LED was switched on at $t=0 \mathrm{~s}$ and the Hall bar was illuminated permanently until $R_{x x}$ passes through $R_{x x \text {, max }}$. The time to reach $R_{x x \text {, max }}$, i.e., $\Delta t$, was recorded for each light power and is depicted in Fig. 4(a). After recording $\Delta t$ for a constant light power, the sample was heated up. After some time at elevated temperatures, the sample returns to its initial state and the next measurement with a different light power was performed. For low light powers, $\Delta t$ is about $100 \mathrm{~s}$ and reduces to $0.6 \mathrm{~s}$ as the light power increases from $0.1 \mathrm{nW}$ to $1 \mu \mathrm{W}$. From the fit function, a slope of -0.58 can be deduced. The previous reported time of $28 \mathrm{~s}$ corresponds to a light power of about $1 \mathrm{nW}$. Please note that the light power values are overestimated. The light of the LED was not focused on the Hall bar under study and only a fraction of the actual emitted light accounts for the optical tunability. Hence, light powers in the picowatt range should be sufficient.

We attribute the optical tunability to the interplay of negative persistent photoconductivity and the buildup of photogenerated charge carriers on the surface and substrate side of the device, respectively. Quantum well heterostructures composed of $6.1 \AA$ family semiconductors exhibit positive and negative persistent photoconductivity, depending on the utilized material, material composition, heterostructure layout, and photon energy [32-40]. In Refs. [33,36,37], the authors study the evolution from positive to negative photoconductivity 

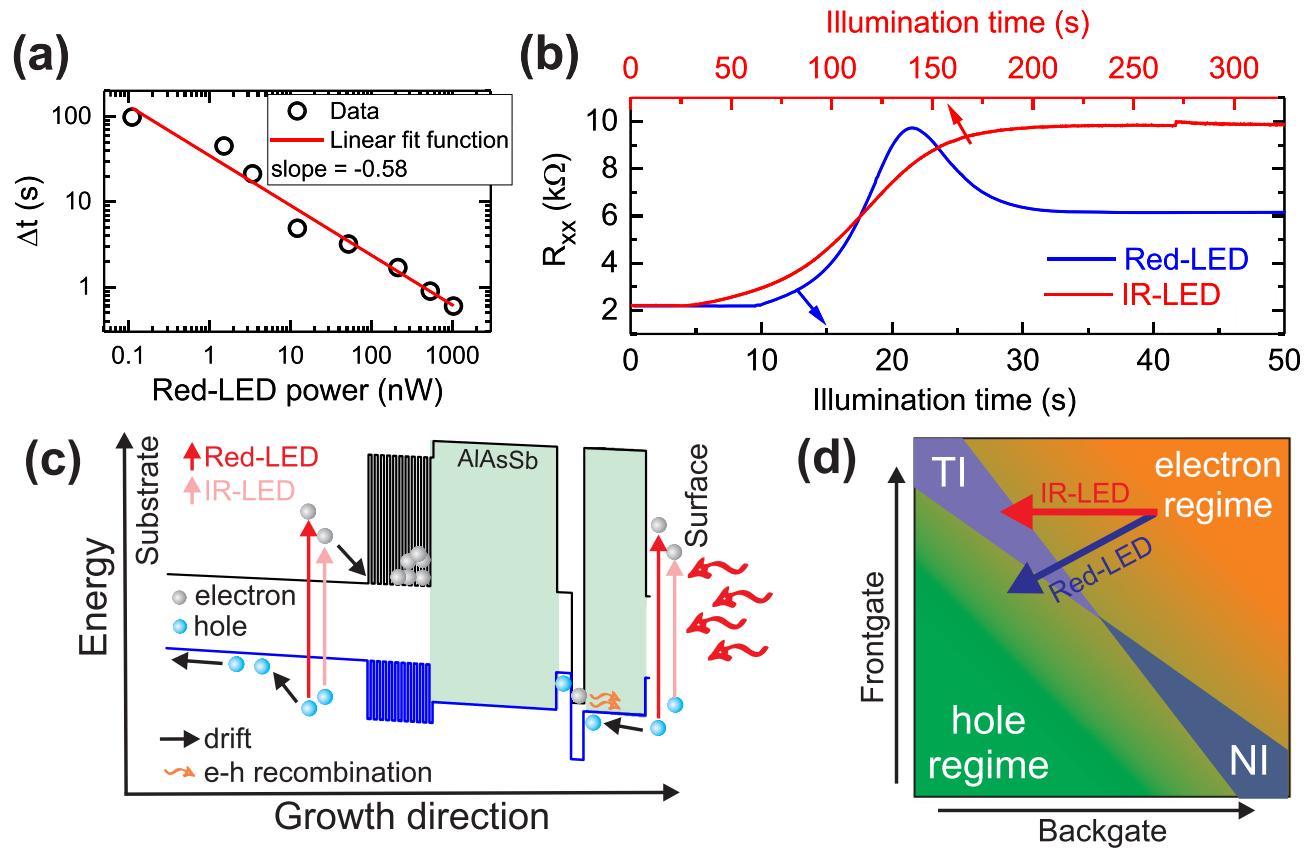

FIG. 4. (a) Illumination time $(\Delta t)$ required to reach $R_{x x, \max }$ as a function of the Red-LED light power. For low light powers, the maximum of the longitudinal resistance is obtained at about $100 \mathrm{~s}$. $\Delta t$ drops to $0.6 \mathrm{~s}$ as the LED power increases by four orders of magnitude. The slope is determined to be -0.58 . (b) Comparison of $R_{x x}$ versus the illumination time for the Red- and IR-LED. Under illumination with the IR-LED, the system switches to $R_{x x \text {, max }}$ at much longer timescales but remains high as the illumination time increases. (c) Scheme of the band profile and the light absorption and accumulation processes involved. At the GaSb cap surface region, illumination with energies above 1.55 eV leads to hole accumulation and successive electron-hole recombination at the InAs quantum well. This process only occurs for the Red-LED. On the backside of the DQW, electron-hole pairs are created for both LEDs, leading to an electron accumulation on the backside of the device. (d) Scheme of the phase diagram of a dual gated InAs/GaSb double quantum well. Via the back and front gate voltage, the system can be switched between the electron regime, the hole regime, the trivial insulating (NI) regime, and the topological insulating regime. In the presented device and under illumination, the Red-LED enables both a front and a back gating of the device, while the IR-LED only enables a back gating of the structure.

of $\mathrm{AlSb} / \mathrm{InAs} / \mathrm{AlSb}$ quantum wells dependent on the incident light energy. For photon energies above $\approx 1.55 \mathrm{eV}$, the authors show negative persistent photoconductivity which they attribute to photogenerated holes in the GaSb cap. At sufficient photon energies, i.e., above $1.55 \mathrm{eV}$, these holes can tunnel through the GaSb/AlSb barrier and accumulate at the InAs quantum well at which they recombine with electrons. This process leads to the depletion of electrons in the InAs quantum well, in analogy to the electric-field tuning via a negative front gate. This negative persistent photoconductivity has been also shown for GaSb/InAs films recently [41]. For lower photon energies, this process is absent (see Ref. [33]) as the hole tunneling time exceeds the recombination time and the photogenerated electron-hole pairs recombine. The electrons are trapped within ionized donors in the GaSb cap region [33].

Figure 4(c) depicts the scheme of this process. To test the hypothesis that photons with energies above and below $1.55 \mathrm{eV}$ enable or disable a negative front gate operation, $R_{x x}$ was also recorded for a second light energy: IR-LED $(1.32 \mathrm{eV})$. Figure 4(b) shows and compares the $R_{x x}$ time traces for both LEDs. Independent of the photon energy, the $R_{x x}$ traces increase over the light exposure time. However, two distinct differences can be observed. First, $R_{x x, \max }$ is reached at much shorter time scales for the Red-LED compared to the IR-LED (about one order of magnitude). The timescale difference can be attributed to the light power difference. Second and more important, $R_{x x}$ remains at $R_{x x, \max }$ for the IR-LED while for the Red-LED, $R_{x x}$ decreases after passing $R_{x x \text {, } \max }$. Hence, while the Red-LED switches the dominant charge carrier type from electron to hole after passing the gap region at $R_{x x \text {, max }}$, the IR-LED trace stops within the gap region. This effect is analogous to a back-gate effect in the electric-field tuning picture. The back-gate operation of the presented device is attributed to electron-hole pair creation at the substrate side of the DQW. Under illumination with photons above the GaSb band-gap energy $E_{\text {gap }} \approx 0.81 \mathrm{eV}$ (true for both LED energies), electron-hole pairs are created on the substrate side of the DQW [see Fig. 4(c)]. A small built-in electric field is sufficient to effectively separate the electron-hole pairs. Electrons can accumulate in the $\mathrm{AlSb} / \mathrm{GaSb}$ superlattice, while holes drift toward the substrate side at which they ultimately recombine. As electron-hole pairs are created via interband absorption, we expect that the back-gate effect can be observed also for IR excitation but at longer timescale due to the reduced absorption coefficient. Deduced control experiments on different sample layouts with different InAs quantum well widths (see Supplemental Material [30]) confirm that the superlattice (SL) on the substrate side is essential to observe the charge carrier switching since without the SL, the back gating action is absent. From these control experiments, we further conclude that $D X$ centers [40] or charge carriers created in the 
InAs or GaSb quantum well do not contribute to the optical switching.

Figure 4(d) shows a scheme of the phase diagram for frontand back-gate control, first calculated by Liu et al. [6], and later shown experimentally in Ref. [14]. In the presented device, the Red-LED enables a back and front gate operation (as indicated by the arrow direction), while the IR-LED only enables backgate operation (also indicated by the arrow direction).

In summary, we studied the optical tunability of the dominant charge carrier type in the topological phase of InAs/GaSb double quantum wells. For low and large illumination densities, the majority charge carrier type is found to be electronand holelike, respectively. Under intermediate illumination densities, electron-hole hybridization occurs and the system switches to a nontrivial topological insulating phase with a gap. The interplay of negative photoconductivity of antimonide materials and the buildup of photogenerated charge carriers, respectively, enables a simultaneous control of the Fermi energy and electric field. The optically induced dual gating of InAs/GaSb double quantum wells amends for leakage currents in these materials and paves the way toward electro-optical devices based on topological materials. In particular, as the switching between normal (NI) to topological (TI) insulating phases requires both gate voltages to change, lateral NI-TI-NI interfaces would require spatially defined front- and back-gate electrodes. With the presented optical tuning, spatially defined back-gate electrodes can be envisioned via shadow masks and do not require selective underetching techniques or thinning of the substrate.

The work was supported by the DFG (Project Ka2318/5-1) and the Elite Network of Bavaria within the graduate program "Topological Insulators." Expert technical assistance by M. Emmerling and A. Wolf is gratefully acknowledged.
[1] C. L. Kane and E. J. Mele, Phys. Rev. Lett. 95, 146802 (2005).

[2] B. A. Bernevig and S.-C. Zhang, Phys. Rev. Lett. 96, 106802 (2006).

[3] B. A. Bernevig, T. L. Hughes, and S.-C. Zhang, Science 314, 1757 (2006).

[4] M. Hasan and C. Kane, Rev. Mod. Phys. 82, 3045 (2010).

[5] M. König, S. Wiedmann, C. Brune, A. Roth, H. Buhmann, L. W. Molenkamp, X.-L. Qi, and S.-C. Zhang, Science 318, 766 (2007).

[6] C. Liu, T. L. Hughes, X.-L. Qi, K. Wang, and S.-C. Zhang, Phys. Rev. Lett. 100, 236601 (2008).

[7] I. Knez, C. T. Rettner, S.-H. Yang, S. S. P. Parkin, L. Du, R.-R. Du, and G. Sullivan, Phys. Rev. Lett. 112, 026602 (2014).

[8] I. Knez, R.-R. Du, and G. Sullivan, Phys. Rev. Lett. 107, 136603 (2011).

[9] I. Knez, R. R. Du, and G. Sullivan, Phys. Rev. B 81, 201301 (2010)

[10] I. Knez, R.-R. Du, and G. Sullivan, Phys. Rev. Lett. 109, 186603 (2012).

[11] F. Nichele, A. N. Pal, P. Pietsch, T. Ihn, K. Ensslin, C. Charpentier, and W. Wegscheider, Phys. Rev. Lett. 112, 036802 (2014).

[12] M. Karalic, S. Mueller, C. Mittag, K. Pakrouski, Q. S. Wu, A. A. Soluyanov, M. Troyer, T. Tschirky, W. Wegscheider, K. Ensslin, and T. Ihn, Phys. Rev. B 94, 241402 (2016).

[13] A. N. Pal, S. Müller, T. Ihn, K. Ensslin, T. Tschirky, C. Charpentier, and W. Wegscheider, AIP Adv. 5, 077106 (2015).

[14] F. Qu, A. J. A. Beukman, S. Nadj-Perge, M. Wimmer, B.-M. Nguyen, W. Yi, J. Thorp, M. Sokolich, A. A. Kiselev, M. J. Manfra, C. M. Marcus, and L. P. Kouwenhoven, Phys. Rev. Lett. 115, 036803 (2015).

[15] E. M. Spanton, K. C. Nowack, L. Du, G. Sullivan, R.-R. Du, and K. A. Moler, Phys. Rev. Lett. 113, 026804 (2014).

[16] K. Suzuki, Y. Harada, K. Onomitsu, and K. Muraki, Phys. Rev. B 87, 235311 (2013).

[17] I. Knez, Transport properties of topological phases in broken gap InAs/GaSb based quantum wells, Ph.D. thesis, Rice University, 2012.
[18] B.-M. Nguyen, A. A. Kiselev, R. Noah, W. Yi, F. Qu, A. J. A. Beukman, F. K. de Vries, J. van Veen, S. Nadj-Perge, L. P. Kouwenhoven, M. Kjaergaard, H. J. Suominen, F. Nichele, C. M. Marcus, M. J. Manfra, and M. Sokolich, Phys. Rev. Lett. 117, 077701 (2016).

[19] L. Du, T. Li, W. Lou, X. Wu, X. Liu, Z. Han, C. Zhang, G. Sullivan, A. Ikhlassi, K. Chang, and R.-R. Du, Phys. Rev. Lett. 119, 056803 (2017).

[20] S. Mueller, A. N. Pal, M. Karalic, T. Tschirky, C. Charpentier, W. Wegscheider, K. Ensslin, and T. Ihn, Phys. Rev. B 92, 081303 (2015).

[21] L. Du, X. Li, W. Lou, G. Sullivan, K. Chang, J. Kono, and R.-R. Du, Nat. Commun. 8, 1971 (2017).

[22] C. Mittag, M. Karalic, S. Mueller, T. Tschirky, W. Wegscheider, O. Nazarenko, M. V Kovalenko, T. Ihn, and K. Ensslin, Appl. Phys. Lett. 111, 082101 (2017).

[23] L. O. Olsson, C. B. M. Andersson, M. C. Håkansson, J. Kanski, L. Ilver, and U. O. Karlsson, Phys. Rev. Lett. 76, 3626 (1996).

[24] M. Noguchi, K. Hirakawa, and T. Ikoma, Phys. Rev. Lett. 66, 2243 (1991).

[25] A. J. Rosenberg, J. Phys. Chem. Solids 14, 175 (1960).

[26] E. Papis-Polakowska, Electron Technol. - Internet J. 38, 1 (2006).

[27] E. A. Plis, M. N. Kutty, and S. Krishna, Laser Photon. Rev. 7, 45 (2013).

[28] B. Nguyen, W. Yi, R. Noah, J. Thorp, and M. Sokolich, Appl. Phys. Lett. 106, 032107 (2015).

[29] C. Nguyen, B. Brar, C. R. Bolognesi, J. J. Pekarik, and H. Kroemer, J. Electron. Mater. 22, 255 (1993).

[30] See Supplemental Material at http://link.aps.org/supplemental/ 10.1103/PhysRevB.98.041301 for details on molecular beam epitaxy fabrication, temperature dependence, and control experiments.

[31] S. Birner, Nextnano++, http://www.Nextnano.com/.

[32] I. Lo, W. C. Mitchel, R. Kaspi, S. Elhamri, and R. S. Newrock, Appl. Phys. Lett. 65, 1024 (1994). 
[33] C. Gauer, J. Scriba, A. Wixforth, J. P. Kotthaus, G. Tuttle, J. H. English, and H. Kroemer, Semicond. Sci. Technol. 8, S137 (1993).

[34] H. Schets, P. Janssen, J. Witters, and S. Borghs, Solid State Commun. 110, 169 (1999).

[35] W. C. Wang, L. C. Tsai, J. C. Fan, Y. F. Chen, and I. Lo, J. Appl. Phys. 86, 3152 (1999).

[36] V. Y. Aleshkin, V. I. Gavrilenko, D. M. Gaponova, A. V. Ikonnikov, K. V. Marem'yanin, S. V. Morozov, Y. G. Sadofyev, S. R. Johnson, and Y.-H. Zhang, Semiconductors 39, 22 (2005).
[37] V. I. Gavrilenko, A. V. Ikonnikov, S. S. Krishtopenko, A. A. Lastovkin, K. V. Marem'yanin, Y. G. Sadofyev, and K. E. Spirin, Semiconductors 44, 616 (2010).

[38] G. Tuttle, H. Kroemer, and J. H. English, J. Appl. Phys. 65, 5239 (1989).

[39] G. Tuttle, H. Kroemer, and J. H. English, J. Appl. Phys. 67, 3032 (1990).

[40] P. M. Mooney, J. Appl. Phys. 67, R1 (1990).

[41] B. Tong, Z. Han, T. Li, C. Zhang, G. Sullivan, and R. R. Du, AIP Adv. 7, 075211 (2017). 